\title{
Hacia el esclarecimiento del rol del anión cloruro en la hipertensión arterial: su vínculo con el daño oxidativo en el riñón
}

\author{
Towards the Elucidation of the Role of the Chloride Anion in Arterial Hypertension: Its Link \\ with Oxidative Damage in the Kidney
}

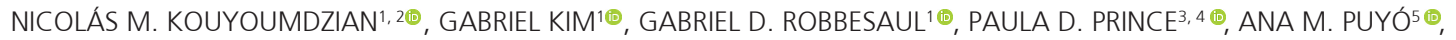 \\ BELISARIO E. FERNÁNDEZ2 ${ }^{\oplus}$, MÓNICA L. GALLEANO ${ }^{3,4}$, MARCELO R. CHOI ${ }^{1,2,5 \odot}$
}

\begin{abstract}
RESUMEN
Introducción: Se desconoce el papel del anión cloruro en los efectos deletéreos del consumo excesivo de sal (NaCl) y si sus efectos son independientes de la presencia del sodio.

Objetivo: Demostrar que tanto una sobrecarga de cloruro como una sobrecarga de sodio en la dieta producen efectos deletéreos, en forma independiente, sobre la presión arterial sistólica (PAS), la función renal y los marcadores de estrés oxidativo en el riñón.

Materiales y métodos: Ratas Wistar macho fueron divididas en cuatro grupos ( $\mathrm{n}=8 /$ grupo) y fueron alimentadas con diferentes dietas durante tres semanas: C: control (dieta estándar), $\mathrm{NaCl}$ : hipersódica-hiperclórica, $\mathrm{Na}$ : hipersódica sin cloruro, Cl: hiperclórica sin sodio. Se determinaron la presión arterial sistólica (PAS) y la función renal y en la corteza renal, se evaluó la producción de especies reactivas del ácido tiobarbitúrico (en inglés: TBARS) y la actividad y la expresión de las enzimas superóxido dismutasa (SOD), catalasa (CAT) y glutatión peroxidasa (GPx).

Resultados: Al cabo de tres semanas, la PAS aumentó (*) en los dos grupos alimentados con cloruro. La excreción fraccional de sodio y de cloruro aumentó (*) en los grupos $\mathrm{NaCl}$ y $\mathrm{Na}$. La diuresis y los TBARS en la corteza renal aumentaron (*) con las tres dietas, sin cambios en la actividad y en la expresión de SOD y CAT. La actividad de la GPx aumentó (*) en los dos grupos que recibieron cloruro; (*p $<0,05$ vs C).

Conclusión: Tanto la sobrecarga de sodio como la de cloruro se asocian a mayor estado oxidativo caracterizado por un incremento en la peroxidación lipídica en la corteza renal. Sin embargo, solo el exceso de cloruro se asocia a mayor actividad de la GPx y de la hipertensión, sin cambios en la excreción urinaria de cloruros, sugiriendo un mayor estado prooxidante renal en comparación con el grupo Na.
\end{abstract}

Palabras clave: Cloruro - Hipertensión - Riñón - Peroxidación de lípido - Glutatión peroxidasa

\begin{abstract}
Introduction: The role of the chloride anion on the deleterious effects of excessive consumption of salt ( $\mathrm{NaCl})$ and whether its effects are independent each other of the presence of sodium remains to date, unknown and unclear.

Objective: To demonstrate that both a chloride overload and a sodium overload in the diet produce deleterious effects, by different mechanisms, on systolic blood pressure (SBP), renal function and markers of oxidative stress in the kidney.

Materials and Methods: Male Wistar rats were divided into four groups ( $\mathrm{n}=8$ / group) and fed with different diets for three weeks: C: control (standard diet), and diets: NaCl: hypersodic-hyperchloric; Na: hypersodic without chloride and Cl: hyperchloric without sodium. Systolic blood pressure (SBP) and renal function were determined, and the production of thiobarbituric acid reactive species (TBARS) and the activity and expression of superoxide dismutase (SOD), catalase (CAT) and glutathione peroxidase (GPx) enzymes were evaluated in renal cortex tissue.

Results: SBP increased (*) in the two groups fed with chloride. The fractional excretion of sodium and chloride increased (*) in the $\mathrm{NaCl}$ and $\mathrm{Na}$ groups. increased (*) in the renal cortex with the three diets. No changes were observed in the activity and expression of SOD and CAT. GPx activity increased (*) in the two groups that received chloride; (* p <0.05 vs C). Conclusion: Both sodium and chloride overload are associated with a higher oxidative state characterized by an increase in lipid peroxidation in the renal cortex. However, compared with Na group, only chloride overload is associated with higher GPx activity and hypertension without any changes in urinary chloride excretion, suggesting a higher renal pro-oxidant state in this experimental group.
\end{abstract}

Key words: Chloride - Hypertension - Kidney - Lipid Peroxidation - Glutathione Peroxidase

Rev Argent CARdiol 2021;89:98-106. http://dx.doi.org/10.7775/rac.es.v89.i2.20034

Recibido: 09/01/2021 - Aceptado: 19/02/2021

Dirección para separatas: Nicolás Martín Kouyoumdzian - CONICET-Universidad de Buenos Aires, Instituto Alberto C. Taquini de Investigaciones en Medicina Traslacional (IATIMET) - Marcelo T. de Alvear 2270 - (C1122AAJ) - CABA, Buenos Aires, Argentina - Tel: (54 11) 4508-3880 - E-mail: nicokouy214@gmail.com

Fuentes de apoyo: El trabajo fue subsidiado por la Agencia Nacional de Promoción Científica y Tecnológica (ANPCYT), la Universidad de Buenos Aires, la Sociedad Argentina de Hipertensión Arterial y la Fundación Héctor A. Barceló.

Este trabajo obtuvo el Premio Braun Menéndez 2020

${ }^{1}$ CONICET-Universidad de Buenos Aires, Instituto Alberto C. Taquini de Investigaciones en Medicina Traslacional (IATIMET). CABA, Argentina ${ }^{2}$ Fundación H.A. Barceló, Instituto Universitario de Ciencias de la Salud. CABA, Argentina

${ }^{3}$ Facultad de Farmacia y Bioquímica, Universidad de Buenos Aires, Cátedra de Fisicoquímica, Departamento de Química Analítica y Fisicoquímica. CABA, Argentina ${ }^{4}$ CONICET-Universidad de Buenos Aires, Instituto de Bioquímica y Medicina Molecular (IBIMOL). CABA, Argentina

${ }^{5}$ Facultad de Farmacia y Bioquímica, Universidad de Buenos Aires, Cátedra de Anatomía e Histología, Departamento de Ciencias Biológicas. CABA, Argentina. 


\section{INTRODUCCIÓN}

El consumo excesivo de sal en la dieta es un factor de riesgo para el desarrollo de hipertensión arterial. En el riñón, la sobrecarga salina induce estrés oxidativo e inflamación, independientemente del valor de la presión arterial. Los estudios clínicos sugieren que la presión arterial no se incrementa por una dieta alta en sodio $\left(\mathrm{Na}^{+}\right)$en ausencia de cloruro $\left(\mathrm{Cl}^{-}\right)$, (1-3) pues el bicarbonato de sodio no tiene el mismo efecto presor que el cloruro de sodio $(\mathrm{NaCl})$ en personas hipertensas. $(2,4)$ Evidencias recientes sugieren que el cloruro puede tener una injerencia más específica en la hipertensión arterial "sal sensible", independiente del efecto hipertensógeno del sodio. (5-9)

Nuestro grupo de trabajo ha demostrado la presencia de efectos proinflamatorios y profibróticos agudos y crónicos que una sobrecarga de $\mathrm{NaCl}$ provoca en el riñón. (10-13) Una dieta alta en $\mathrm{NaCl}$ induce activación del gen del angiotensinógeno, mayor síntesis de angiotensina II renal y aumento del estrés oxidativo que conducen al desarrollo de hipertensión. (14-16) Sin embargo, hasta el presente, no están descriptos ni esclarecidos los posibles efectos dañinos de una sobrecarga del $\mathrm{Cl}^{-}$en el riñón, y si sus efectos son independientes de la presencia del $\mathrm{Na}^{+}$.

\section{Hipótesis}

El anión cloruro, independientemente del catión sodio, también estaría involucrado en el estrés oxidativo del riñón y la elevación de la presión arterial. Estas alteraciones se verían atenuadas si el cloruro se reemplazara por otro anión (por ejemplo, el citrato) o si el sodio fuese reemplazado por otros cationes.

\section{Objetivos}

Discernir cuáles son los efectos propios de la sobrecarga de cloruro en la dieta y cuáles los de la sobrecarga de sodio sobre los siguientes parámetros:

- presión arterial sistólica (PAS)

- función renal

- marcadores de estrés oxidativo en el riñón

\section{MATERIALES Y MÉTODOS}

\section{Animales utilizados}

Se utilizaron 32 ratas Wistar macho de 7 semanas de edad y peso corporal (PC) promedio de 155-165 g al comienzo de la dieta.

\section{Dietas}

Los animales se dividieron en un grupo control y tres grupos experimentales ( $\mathrm{n}=8$ /grupo), bebieron agua corriente $a d$ libitum, y consumieron esas dietas (17) durante 3 semanas:

1) Control: dieta estándar $(0,4 \% \mathrm{P} / \mathrm{P}$ de $\mathrm{NaCl}$ en la comida)

2) $\mathrm{NaCl}$ : dieta alta en cloruro de sodio $(8 \%)$

3) Na: dieta hipersódica sin cloruro $\left(\mathrm{Na}_{3} \mathrm{C}_{6} \mathrm{H}_{5} \mathrm{O}_{7} 11,8 \%\right)$ (equimolar en $\mathrm{Na}^{+}$con grupo 2)

4) $\boldsymbol{C l}$ : dieta hiperclórica sin sodio $\left(\mathrm{CaCl}_{2} 3,80 \% ; \mathrm{KCl} 3,06 \%\right.$ y $\mathrm{MgCl}_{2} 1,30 \%$ ) (equimolar en $\mathrm{Cl}^{-}$con grupo 2 ).
Determinación de la presión arterial sistólica

Se midió la PAS a tiempo 0 (basal), 1, 2 y 3 semanas por esfingomanometría en la cola de las ratas (Hatteras Instruments, Cary, NC, US), entre las 9 y 11 horas, previo entrenamiento de los animales durante 3 días consecutivos.

\section{Determinación del consumo de alimento, ingesta calórica y consumo de bebida}

Durante tres días consecutivos de la tercera semana, se calcularon los consumos de alimento $(\mathrm{g})$ y agua $(\mathrm{mL})$. Se calculó la ingesta calórica (kcal) como: $3,3 \mathrm{kcal} / \mathrm{g} *$ Ingesta de alimento $(\mathrm{g})$.

\section{Determinación de parámetros urinarios, plasmáticos y evaluación de la funcionalidad renal excretora}

$\mathrm{Al}$ cabo de las 3 semanas de dieta, los animales se alojaron en jaulas metabólicas, durante dos días: uno de aclimatación y otro de recolección de orina de 24 horas para medir la diuresis, las concentraciones urinarias de $\mathrm{Na}^{+}$y $\mathrm{Cl}^{-}(\mathrm{mEq} / \mathrm{L})$ y creatinina $(\mathrm{mg} / \mathrm{dL})$.

El día del sacrificio, luego de obtener el peso corporal final, y bajo anestesia con ketamina $(60 \mathrm{mg} / \mathrm{kg}$ ) y xilacina $(2 \mathrm{mg} / \mathrm{kg})$, se extrajo sangre del seno retroocular. Se determinaron las concentraciones plasmáticas de $\mathrm{Na}^{+}, \mathrm{Cl}$, creatinina, glucosa y urea, mediante un autoanalizador. La osmolaridad plasmática $(\mathrm{mOsm} / \mathrm{kg})$ se estimó como:

$2 *$ natremia $(\mathrm{mEq} / \mathrm{L})+1 / 18^{*}$ glucemia $(\mathrm{mg} / \mathrm{dL})+1 / 6 *$ uremia $(\mathrm{mg} / \mathrm{dL})$.

Se calculó la depuración (clearance) de creatinina según: $\mathrm{ClCr}=(\text { creatininuria/creatininemia })^{*}$ diuresis/tiempo/PC.

Para estudiar la función tubular se calcularon la carga filtrada (CF), la excreción urinaria (EU), la excreción fraccional (EF), la reabsorción tubular (RT) y la reabsorción fraccional (RF) de los diferentes iones, a partir de las siguientes fórmulas estándares:

$$
\begin{aligned}
& \mathrm{CFNa}=\mathrm{ClCr} * \text { natremia } \quad \mathrm{EUNa}=\text { diuresis*natruria } \\
& \mathrm{RTNa}=\mathrm{CFNa}-\mathrm{EUNa} \quad \mathrm{EFNa}=(\mathrm{EUNa} / \mathrm{CFNa}) * 100 \\
& \mathrm{CFCl}=\mathrm{ClCr} * \text { cloremia } \quad \mathrm{EUCl}=\text { diuresis } * \text { cloruria } \\
& \mathrm{RTCl}=\mathrm{CFCl}-\mathrm{EUCl} \quad \mathrm{EFCl}=(\mathrm{EUCl} / \mathrm{CFCl}) * 100
\end{aligned}
$$

La diuresis, ClCr, y CF, RT y EU se normalizaron por el PC de cada rata y se expresan en $\mathrm{mL} /$ día/kg, $\mathrm{mL} / \mathrm{min} / \mathrm{kg}$ o $\mathrm{mEq} /$ día/kg, mientras que la EF y RF se expresan en porcentaje (\%).

\section{Sacrificio, extracción de riñones y procesamiento de muestras}

Bajo anestesia, se extrajeron mediante laparatomía abdominal ambos riñones. Se disecó la corteza renal, que se homogenizó en Buffer Fosfato Salino (7,6 mM KH $\mathrm{PO}_{4}, 42,4 \mathrm{mM} \mathrm{K}_{2} \mathrm{HPO}_{4}$, $150 \mathrm{mM} \mathrm{NaCl}, \mathrm{pH}: 7,4)$ y se centrifugó a $600 \mathrm{~g}$ durante 20 minutos a $4{ }^{\circ} \mathrm{C}$. En los sobrenadantes se determinaron las especies reactivas del ácido tiobarbitúrico (TBARS), y la actividad de las enzimas antioxidantes: superóxido dismutasa (SOD), catalasa (CAT) y glutatión peroxidasa (GPx). La expresión proteica de estas enzimas se determinó mediante Western Blot en homogenatos de corteza renal. El contenido proteico se midió por el método de Lowry. (18) Los animales fueron sacrificados por decapitación.

\section{Determinación de TBARS}

El ensayo se basa en un método previamente descripto por Fraga y col. (19) Los resultados se expresan en nmol de TBARS de equivalentes de malondialdehído (MDA)/mg proteína.

\section{Determinación de actividades enzimáticas}

La actividad de la SOD fue medida espectrofotométricamente siguiendo la formación del adenocromo. En ausencia de KCN 
( $4 \mu \mathrm{M}$ ) se determinó la actividad total de SOD; en su presencia, la actividad de Mn-SOD (isoforma mitocondrial); y por diferencia, la actividad de $\mathrm{Cu} / \mathrm{Zn}$-SOD (isoforma citosólica). Los resultados se expresan en unidades arbitrarias (UA)/mg proteína. (20)

La actividad de la CAT se determinó por espectrofotometría siguiendo el consumo de $\mathrm{H}_{2} \mathrm{O}_{2}$ a $240 \mathrm{~nm}$. Los resultados se expresan como $\mu \mathrm{mol} \mathrm{H}_{2} \mathrm{O}_{2} / \mathrm{mg}$ proteína/min. $(21,22)$

La actividad de la GPx se midió por espectrofotometría siguiendo la oxidación enzimática de NADPH a $340 \mathrm{~nm}$ en presencia de $1 \mathrm{~mm}$ de glutatión (GSH), $1 \mathrm{~mm} \mathrm{NaN}_{3}, 0,15 \mathrm{~mm}$ $\mathrm{NADPH}$ y 0,25 unidades $(\mathrm{U}) / \mathrm{mL}$ de glutatión reductasa. Los resultados se expresan en $\mu$ mol NADPH oxidado/mg proteína/ min, que equivalen a $\mu \mathrm{mol}$ de glutatión oxidado (GSSG)/mg proteína/min). (23)

\section{Determinaciones por Western Blot}

La metodología se describe en trabajos previos. (24) Los anticuerpos primarios se diluyeron 1:1000, y los secundarios, conjugados con peroxidasa de rabanito, 1:5000 en buffer fosfato salino (PBS). Como control de carga se utilizó $\beta$-actina para normalizar el contenido proteico.

\section{Análisis estadístico}

Los resultados se expresan como la media \pm error estándar de la media (EEM). Se realizó un análisis de la varianza (ANOVA) de dos vías y, el test de Tukey a posteriori, empleando el programa InfoStat, versión 2018. Los resultados con valor $\mathrm{p}$ $<0,05$ se consideraron significativos.

\section{Consideraciones éticas}

El protocolo experimental utilizado fue aprobado por el Comité Institucional para el Cuidado y Uso de Animales de Laboratorio de la Facultad de Farmacia y Bioquímica de la Universidad de Buenos Aires (CICUAL), por Res(D) $\mathrm{N}^{\circ} 1881 / 2019$, y los procedimientos fueron realizados siguiendo las indicaciones de la "Guía para el cuidado y uso de animales de laboratorio" perteneciente a la Academia Nacional de Ciencias de los Estados Unidos de Norteamérica.

\section{RESULTADOS}

Peso corporal, ingesta de alimento, calorías y agua Los tres grupos alimentados con dietas experimentales mostraron una diferencia de peso corporal final-inicial menor que los controles, acompañada de mayor ingesta de bebida al cabo de las tres semanas de tratamiento dietario (Tabla 1).

\section{Evolución temporal de la presión arterial sistólica}

Las ratas controles se mantuvieron normotensas durante las 3 semanas de dieta. La PAS aumentó en los tres grupos alimentados con dietas experimentales a partir de la segunda semana; las diferencias fueron significativas con respecto a los valores basales y del grupo control para las dietas $\mathrm{NaCl}$ y $\mathrm{Cl}$.

Los valores de PAS más altos se alcanzaron a las 2 y 3 semanas en el grupo $\mathrm{NaCl}$, mientras que los incrementos de la PAS en los grupos $\mathrm{Cl}$ y $\mathrm{Na}$ fueron menores que los alcanzados en el grupo $\mathrm{NaCl}$. Tal como se puede observar en la Figura 1, la PAS en el grupo Na mostró una elevación menor que en los otros dos grupos experimentales, pero sin alcanzar diferencias significativas con respecto al grupo control (Figura 1).

\section{Parámetros plasmáticos y urinarios}

No se modificó la creatininemia, natremia, cloremia y osmolaridad plasmática (estimada a partir de la natremia, la glucemia y la uremia) en ninguno de los grupos. Con respecto al grupo control, la creatininuria disminuyó en los tres grupos, y la natruria aumentó en los grupos con dieta alta en sodio $(\mathrm{NaCl}$ y $\mathrm{Na}$ ) y disminuyó en el grupo $\mathrm{Cl}$. El índice natruria/cloruria, que evalúa la equimolaridad urinaria entre los dos iones, aumentó significativamente en el grupo $\mathrm{Na}, \mathrm{y}$ alcanzó valores muy cercanos a la equimolaridad en el grupo $\mathrm{Cl}$ (Tabla 2).

\section{Parámetros de funcionalidad renal excretora}

La diuresis aumentó en los tres grupos con respecto al control, mientras que el ClCr disminuyó en los grupos $\mathrm{NaCl}$ y $\mathrm{Na}$.

En los grupos $\mathrm{NaCl}$ y $\mathrm{Na}$ disminuyeron la $\mathrm{CFNa}$, la RTNa, la RFNa, la CFCl, la RTCl y la RFCl y aumentaron la EUNa, la EFNa, la EUCl y la EFCl respecto al control.

En comparación con el grupo $\mathrm{NaCl}$, en las ratas $\mathrm{Na}$ observamos aumento de la EUNa y disminución de la EUCl. Asimismo, en el grupo $\mathrm{Na}$, la $\mathrm{EFCl}$ fue menor que la EFNa, mientras que la RFCl fue mayor que la RFNa.

\begin{tabular}{|lcccc|} 
& Control & NaCl & Na & Cl \\
\hline Número de casos $(\mathrm{n})$ & 8 & 8 & 8 & 8 \\
\hline Peso corporal inicial $(\mathrm{g})$ & $152 \pm 4$ & $151 \pm 6$ & $156 \pm 10$ & $175 \pm 6$ \\
\hline Peso corporal final $(\mathrm{g})$ & $293 \pm 22$ & $265 \pm 21$ & $247 \pm 15$ & $290 \pm 13$ \\
\hline Diferencia de peso corporal $(\mathrm{g})$ & $141 \pm 7$ & $114 \pm 9^{*}$ & $91 \pm 15^{*}$ & $115 \pm 3^{*}$ \\
\hline Ingesta de comida estimada $(\mathrm{g})$ & $27 \pm 2$ & $29 \pm 3$ & $35 \pm 8$ & $33 \pm 3$ \\
\hline Ingesta calórica estimada (kcal) & $91 \pm 7$ & $95 \pm 9$ & $116 \pm 26$ & $110 \pm 10$ \\
\hline Ingesta de bebida estimada $(\mathrm{mL})$ & $21 \pm 1$ & $50 \pm 4$ * & $61 \pm 9^{*}$ & $31 \pm 1^{*}$ \\
\hline
\end{tabular}

$\mathrm{NaCl}$ : dieta hipersódica e hiperclórica; Na: dieta hipersódica sin cloruro; Cl: dieta hiperclórica sin sodio. * $\mathrm{p}<0,05$ con respecto al grupo control.

Tabla 1. Peso corporal, ingesta de comida, calorías y bebida 
Fig. 1. Evolución temporal de la presión arterial sistólica

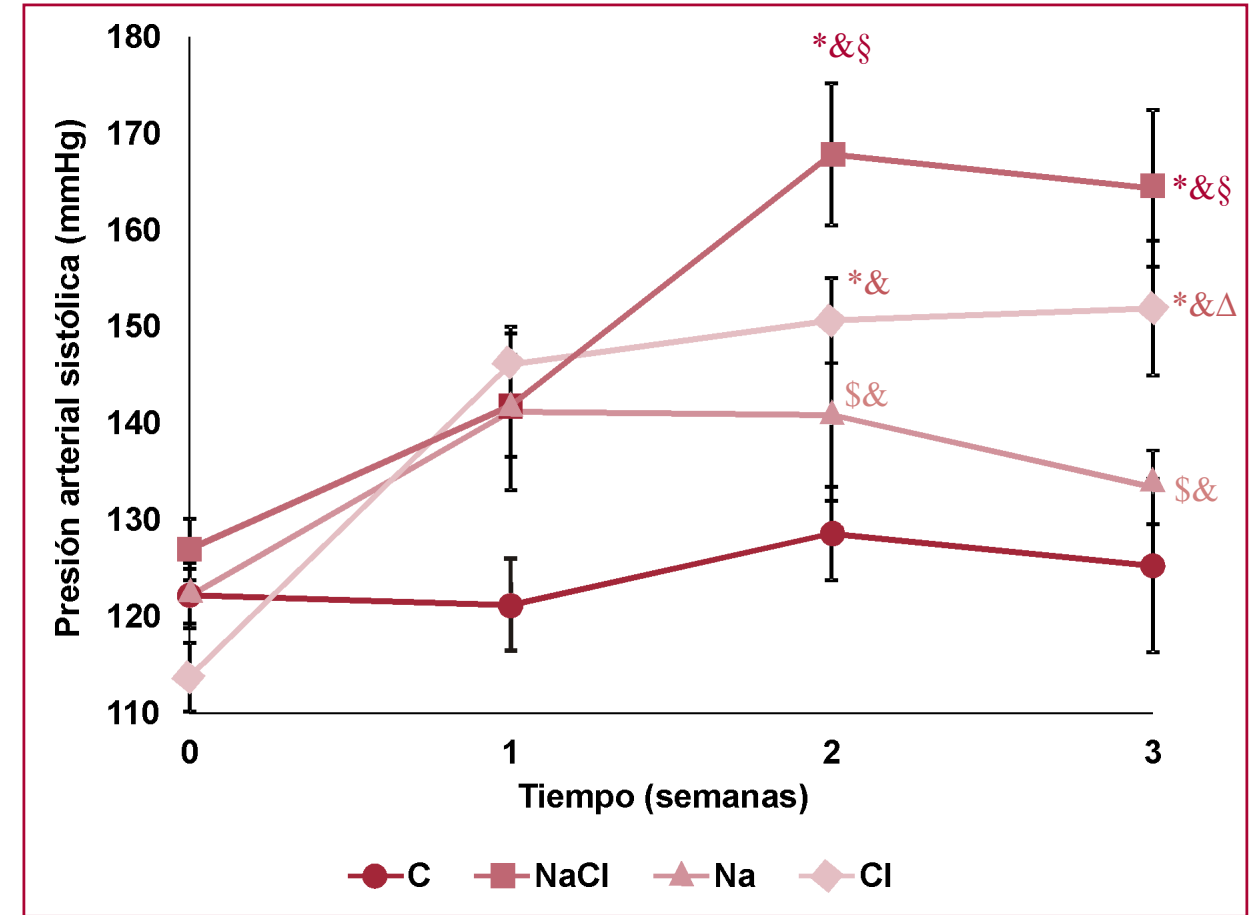

$\mathrm{NaCl}$ : dieta hipersódica e hiperclórica; Na: dieta hipersódica sin cloruro; Cl: dieta hiperclórica sin sodio. $\mathrm{P}<0,05$ : * vs Control; \$ vs $\mathrm{NaCl} ; \Delta$ vs $\mathrm{Na} ; \&$ vs $\mathrm{t}=0 ; \S$ vs $1^{\text {at }}$ semana.
Tabla 2. Parámetros plasmáticos y urinarios

\begin{tabular}{|lcccc|}
\hline & Control & NaCl & Na & Cl \\
\hline Creatininemia $(\mathrm{mg} / \mathrm{dL})$ & $0,56 \pm 0,04$ & $0,64 \pm 0,04$ & $0,62 \pm 0,03$ & $0,63 \pm 0,04$ \\
\hline Natremia $(\mathrm{mEq} / \mathrm{L})$ & $151 \pm 5$ & $144 \pm 2$ & $147 \pm 3$ & $144 \pm 2$ \\
\hline Cloremia $(\mathrm{mEq} / \mathrm{L})$ & $102 \pm 2$ & $100 \pm 1$ & $101 \pm 3$ & $99 \pm 1$ \\
\hline Glucemia $(\mathrm{mg} / \mathrm{dL})$ & $138 \pm 11$ & $152 \pm 15$ & $153 \pm 13$ & $151 \pm 14$ \\
\hline Uremia $(\mathrm{mg} / \mathrm{dL})$ & $27 \pm 1$ & $38 \pm 4^{*}$ & $49 \pm 4 * \$$ & $22 \pm 2 * \$ \Delta$ \\
\hline Osmolaridad plasmática & $319 \pm 9$ & $311 \pm 4$ & $321 \pm 7$ & $306 \pm 5$ \\
\hline estimada $(\mathrm{mOsm} / \mathrm{kg})$ & & & & \\
\hline Creatininuria $(\mathrm{mg} / \mathrm{dL})$ & $316 \pm 42$ & $52 \pm 17^{*}$ & $22 \pm 4^{*}$ & $71 \pm 24 * \Delta$ \\
\hline Natriuria $(\mathrm{mEq} / \mathrm{L})$ & $117 \pm 31$ & $293 \pm 41^{*}$ & $360 \pm 43^{*}$ & $26 \pm 11^{*} \$ \Delta$ \\
\hline Cloruria $(\mathrm{mEq} / \mathrm{L})$ & $145 \pm 37$ & $345 \pm 48^{*}$ & $83 \pm 8 * \$$ & $29 \pm 12^{*} \$ \Delta$ \\
\hline Índice urinario Na $/ \mathrm{Cl}-$ & $0,77 \pm 0,09$ & $0,84 \pm 0,05$ & $4,30 \pm 0,23 * \$$ & $0,99 \pm 0,26 \Delta$ \\
\hline
\end{tabular}

$\mathrm{NaCl}$ : dieta hipersódica e hiperclórica; $\mathrm{Na}$ : dieta hipersódica sin cloruro; Cl: dieta hiperclórica sin sodio. ${ }^{*} \mathrm{p}<0,05$ vs Control; \$ $\mathrm{p}<0,05$ vs $\mathrm{NaCl} ; \Delta \mathrm{p}<0,05$ vs $\mathrm{Na}$.
El grupo $\mathrm{Cl}$ no mostró cambios significativos con respecto al grupo control, pero sí al compararlo con los otros dos grupos: respecto al grupo $\mathrm{NaCl}$, tuvo mayor CFNa, RTNa, RFNa, CFCl, RTCl y RFCl, cambios que se acompañaron por una menor excreción urinaria y fraccional de ambos iones. Con respecto al grupo $\mathrm{Na}$, el grupo $\mathrm{Cl}$ tuvo menor excreción urinaria y fraccional, y mayor reabsorción fraccional de ambos iones (Tabla 3).

\section{Parámetros de estrés oxidativo en corteza renal} La producción de TBARS aumentó en la corteza renal en los grupos $\mathrm{NaCl}$, $\mathrm{Na}$ y $\mathrm{Cl}$ respecto al grupo control.
No se modificaron la actividad y la expresión proteica de la isoforma mitocondrial y citosólica de la SOD y de la CAT. Mientras que la expresión proteica de la GPx no se modificó en ningún grupo, la actividad de esta enzima se incrementó en los grupos $\mathrm{NaCl}$ y $\mathrm{Cl}$ con respecto a los grupos control y $\mathrm{Na}$ (Figura 2 ).

\section{Resumen de resultados}

Tanto el exceso de una dieta alta en sodio como en cloruro se asocian a un mayor estado oxidativo evidenciado por un incremento en la peroxidación lipídica en la corteza renal, demostrado por un incremento en la 


\begin{tabular}{|c|c|c|c|c|}
\hline & Control & $\mathrm{NaCl}$ & $\mathrm{Na}$ & $\mathrm{Cl}$ \\
\hline Diuresis (mL/día/kg) & $10 \pm 2$ & $78 \pm 14^{*}$ & $92 \pm 15^{*}$ & $51 \pm 21 * \Delta$ \\
\hline $\mathrm{ClCr}(\mathrm{mL} / \mathrm{min} / \mathrm{kg})$ & $3,55 \pm 0,55$ & $2,21 \pm 0,29 *$ & $2,41 \pm 0,19 *$ & $3,01 \pm 0,53$ \\
\hline CFNa (mEq/día/kg) & $790 \pm 141$ & $461 \pm 61$ * & $511 \pm 39 *$ & $634 \pm 105 \$$ \\
\hline EUNa (mEq/día/kg) & $1,2 \pm 0,3$ & $22,9 \pm 4,3^{*}$ & $34,4 \pm 6,2 * \$$ & $1,1 \pm 0,3 \$ \Delta$ \\
\hline EFNa (\%) & $0,15 \pm 0,04$ & $5,24 \pm 1,74^{*}$ & $6,82 \pm 0,97^{*}$ & $0,15 \pm 0,03 \$ \Delta$ \\
\hline RTNa (mEq/día/kg) & $789 \pm 141$ & $440 \pm 64^{*}$ & $477 \pm 38^{*}$ & $633 \pm 105 \$$ \\
\hline RFNa (\%) & $99,85 \pm 0,04$ & $94,76 \pm 1,74^{*}$ & $93,18 \pm 0,97^{*}$ & $99,85 \pm 0,03 \$ \Delta$ \\
\hline CFCI (mEq/día/kg) & $532 \pm 92$ & $319 \pm 40 *$ & $349 \pm 26^{*}$ & $435 \pm 74 \$$ \\
\hline EUCI (mEq/día/kg) & $1,4 \pm 0,3$ & $26,5 \pm 5,1^{*}$ & $7,8 \pm 1,5 * \$$ & $1,1 \pm 0,3 \$ \Delta$ \\
\hline $\mathrm{EFCl}(\%)$ & $0,27 \pm 0,07$ & $8,39 \pm 2,70^{*}$ & $2,23 \pm 0,33 * \$ @$ & $0,24 \pm 0,04 \$ \Delta$ \\
\hline RTCI (mEq/día/kg) & $531 \pm 92$ & $295 \pm 44^{*}$ & $341 \pm 26^{*}$ & $434 \pm 74 \$$ \\
\hline $\operatorname{RFCl}(\%)$ & $99,73 \pm 0,07$ & $91,61 \pm 2,70$ * & $97,77 \pm 0,33 * \$ @$ & $99,76 \pm 0,04 \$ \Delta$ \\
\hline
\end{tabular}

$\mathrm{NaCl}$ : dieta hipersódica e hiperclórica; $\mathrm{Na}$ : dieta hipersódica sin cloruro; Cl: dieta hiperclórica sin sodio. ClCr: clearance de creatinina, CF: carga filtrada, EU: excreción urinaria, EF: excreción fraccional, RT: reabsorción tubular, RF: reabsorción fraccional. * $p<0,05$ vs Control; $\$ p<0,05$ vs NaCl; @p $<0,05$ vs EFNa o RFNa; $\Delta p<0,05$ vs Na.

producción de TBARS. Sin embargo, solo el exceso de cloruro, comparado con el grupo $\mathrm{Na}$, se asocia a actividad mayor de la GPx y al desarrollo de hipertensión con mayor retención urinaria de ambos iones; sugiere un mayor estado prooxidante y de estrés oxidativo en el riñón en presencia de una sobrecarga de cloruro.

\section{DISCUSIÓN}

Peso corporal, ingesta de alimento, calorías y agua La ingesta de $\mathrm{Na}^{+}, \mathrm{Cl}$, o ambos iones se asoció con menor ganancia de $\mathrm{PC}$, respecto al grupo control, durante las tres semanas de dieta. Estos resultados son consistentes con lo reportado en la bibliografía, donde se demostró que una dieta hipersalina se asocia con disminución de la masa grasa total en los ratones que presentaron up regulation de genes involucrados en la lipólisis y down regulation de genes relacionados a la lipogénesis. (25) En nuestro trabajo observamos en el momento del sacrificio, una disminución en la grasa epididimal y perirrenal en las ratas que consumieron $\mathrm{NaCl}$ y $\mathrm{Na}$ respecto a los otros dos grupos experimentales (datos no incluidos). Estos hallazgos ocurrieron a pesar de que todas las dietas fueron isocalóricas entre sí. Los animales alimentados con dietas con sobrecarga de sales consumieron más agua que los controles. Esto puede ser causado por un incremento agudo inicial de la osmolaridad plasmática, que estimula el centro de la sed, con el objeto de compensar ese incremento. (26)

\section{Evolución temporal de la presión arterial sistólica}

Hemos demostrado que ratas Sprague Dawley macho, sometidas a una dieta con sobrecarga de $\mathrm{NaCl}(8 \% \mathrm{P} / \mathrm{P})$ aumentan la PAS al cabo de tres semanas de dieta, con valores que superan los definidos como hipertensión sistólica (140 mmHg). (27)
Los resultados que se presentan sugieren que el aumento de la PAS está también relacionado con la sobrecarga de cloruros, pues el grupo $\mathrm{Cl}$ alcanzó valores tensionales mayores a $140 \mathrm{mmHg}$, superiores a los del grupo $\mathrm{Na}$. El anión $\mathrm{Cl}^{-}$es un componente del $\mathrm{NaCl}$ que podría tener un rol más específico en la sal-sensibilidad y que podría ser, incluso, más determinante que el $\mathrm{Na}^{+}$. (28) Otros estudios efectuados en ratas Dahl "sal-sensibles" mostraron que a lo largo de varias semanas, la hipertensión se desarrolló en los animales consumidores de $\mathrm{NaCl}$, pero no en los alimentados con $\mathrm{NaHCO}_{3}$ u otras sales de $\mathrm{Na}^{+} .(29-31)$

Por otra parte, la sobreingesta de sales de cloruro "no sódicas", que es acompañada por una excreción urinaria de cloruro menor que la producida en presencia de $\mathrm{Na}^{+}$, podría estar relacionada con una acumulación selectiva de $\mathrm{Cl}^{-}$en el organismo, lo que conllevaría al desarrollo de hipertensión arterial "sal-sensible". (32-34)

\section{Parámetros plasmáticos y urinarios}

La ausencia de modificaciones en las concentraciones plasmáticas de sodio y cloruro y la osmolaridad son evidencias de la eficiencia biológica de los mecanismos fisiológicos para compensar una posible hipernatremia y/o hipercloremia y para conservar la osmolaridad plasmática.

Como era esperable, en el grupo $\mathrm{NaCl}$ aumentaron la natruria y la cloruria con respecto al grupo control; el índice urinario $\mathrm{Na}^{+} / \mathrm{Cl}^{-}$fue similar en ambos grupos. En el grupo Na es posible que aumente la secreción y la excreción de bicarbonato, resultado consistente con el incremento del índice urinario $\mathrm{Na}^{+} / \mathrm{Cl}^{-}$con respecto a los demás grupos que observamos; sugiere que el $\mathrm{Cl}^{-}$no es el principal contraión del $\mathrm{Na}^{+}$excretado. La secreción de $\mathrm{HCO}_{3}$ tiene por objeto compensar una alcalosis metabólica en los animales que recibieron citrato de 
Fig. 2. Parámetros de estrés oxidativo en corteza renal
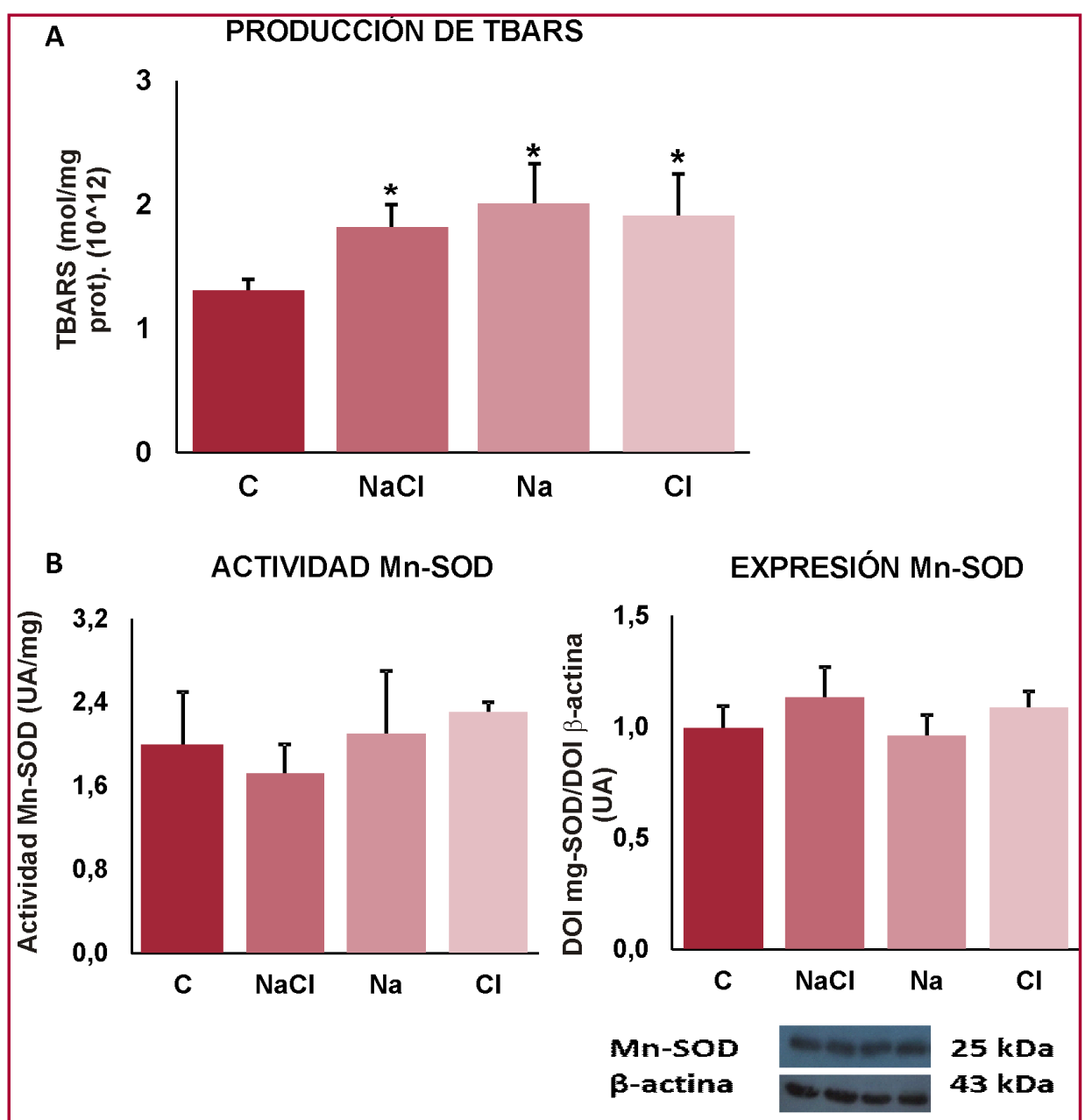

C ACTIVIDAD Cu/Zn-SOD
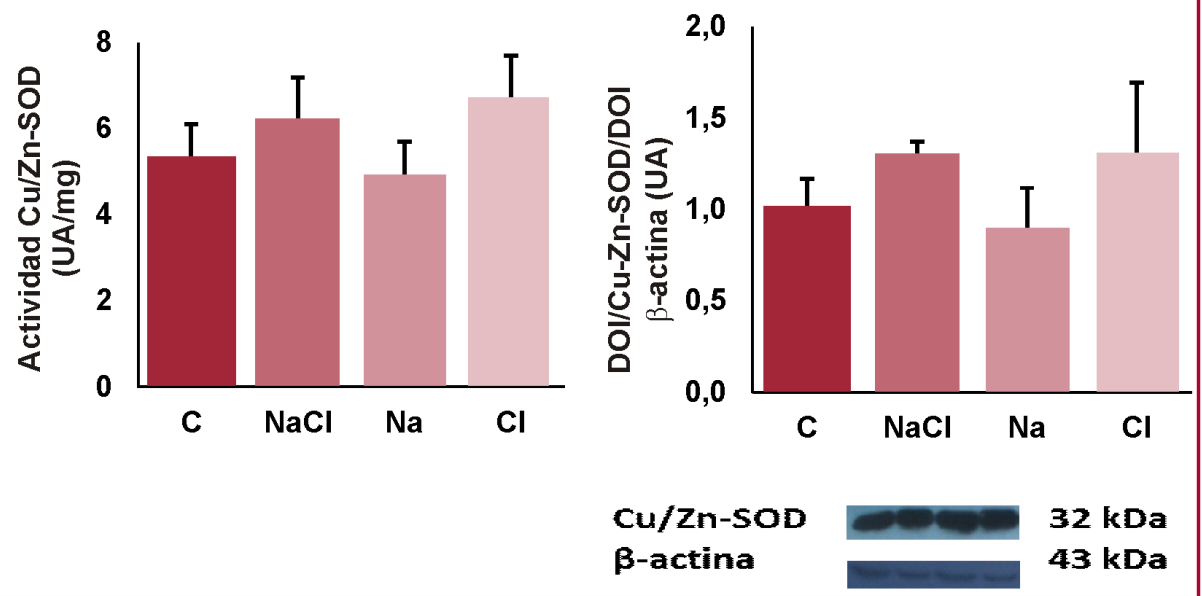


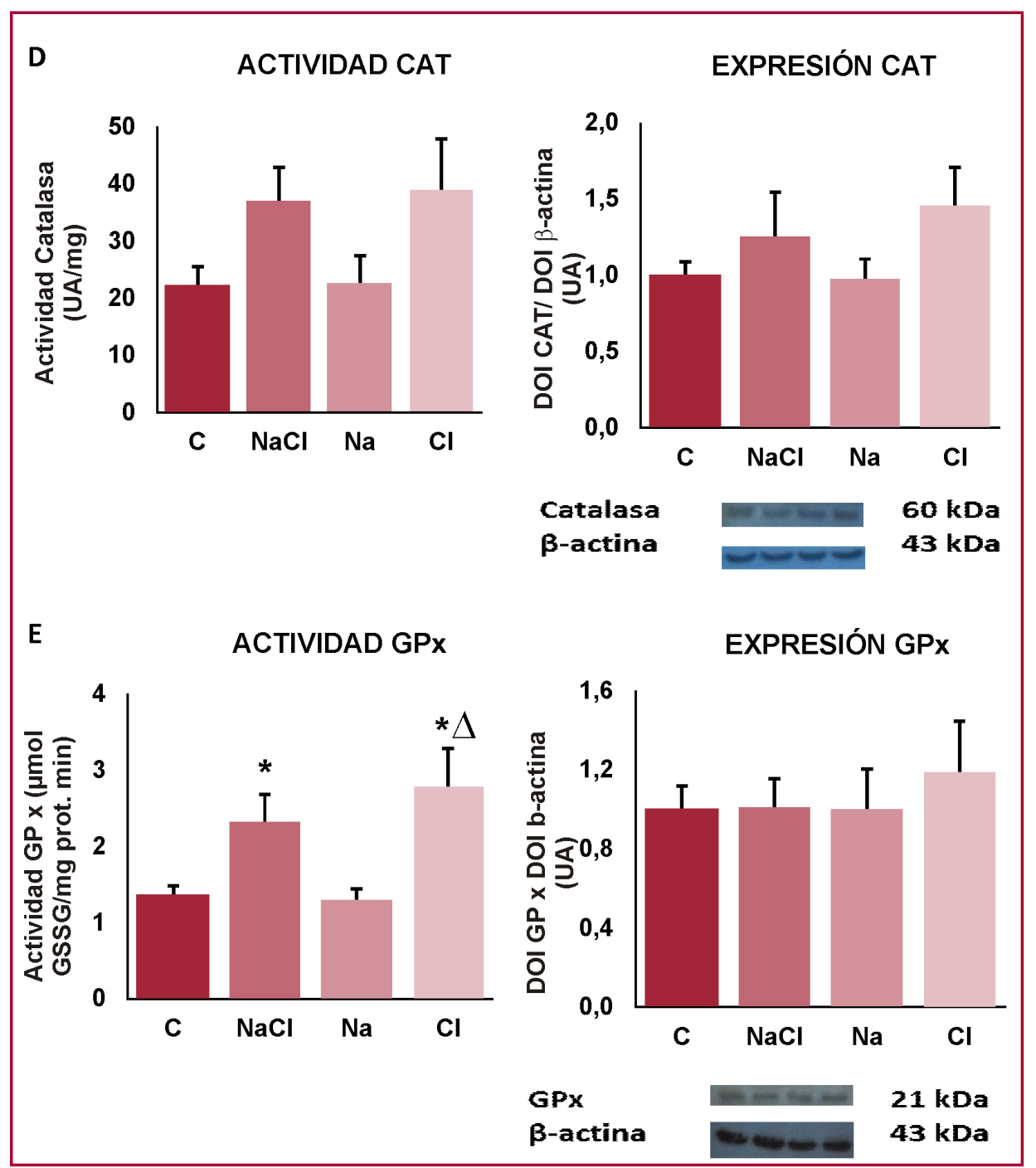

A) TBARS: especies reactivas del ácido tiobarbitúrico. B) Mn-SOD: Manganeso-Superóxido Dismutasa (isoforma mitocondrial de la enzima). C) Cu/Zn-SOD: Cobre/Zinc-Superóxido Dismutasa (isoforma citosólica de la enzima). D) CAT: Catalasa. E) GPx: Glutatión Peroxidasa. $\mathrm{NaCl}$ : dieta hipersódica e hiperclórica; $\mathrm{Na}$ : dieta hipersódica sin cloruro; Cl: dieta hiperclórica sin sodio. * $\mathrm{p}<0,05$ vs Control; $\Delta \mathrm{p}<0,05$ vs Na.

$\mathrm{Na}^{+}$y, como consecuencia de ello, la reabsorción de $\mathrm{Cl}^{-}$estaría incrementada y su excreción, disminuida, ya que el intercambiador $\mathrm{Cl}^{-} / \mathrm{HCO}_{3}^{-}$, independiente del catión $\mathrm{Na}^{+}$, presentaría una mayor expresión en las membranas apicales de las células de los túbulos contorneado distal, colector cortical y conector. (28) En cuanto al grupo $\mathrm{Cl}$ es llamativa la baja cloruria con respecto a las ratas control, lo que sugiere la hipótesis que para su excreción, es necesario que se elimine también el $\mathrm{Na}^{+}$como contraión.

Estos resultados sugieren que el anión cloruro se estaría acumulando en algún compartimento, como la piel, ya que en el plasma sus niveles siguen siendo normales. (32-34)

Parámetros de funcionalidad renal excretora

$\mathrm{El}$ incremento de la diuresis en los tres grupos experimentales, con respecto a los controles, concuerda con el incremento del consumo de bebida. Di Ciano y (continuación) 
Pero ese perfil no se observó en el grupo Cl; demuestra que el reemplazo del ion $\mathrm{Na}^{+}$por otros cationes provoca respuestas disímiles. En el grupo $\mathrm{Cl}$, los iones $\mathrm{Na}^{+}$y $\mathrm{Cl}$ - presentan perfiles de excreción y reabsorción muy similares entre sí, se observa una clara equimolaridad urinaria que refleja que el contraión que se elimina con el $\mathrm{Cl}^{-}$es el $\mathrm{Na}^{+}$.

\section{Parámetros de estrés oxidativo en corteza renal}

La sobrecarga de $\mathrm{Na}^{+}, \mathrm{Cl}^{-}$o ambos iones en la dieta, se asoció con un incremento en la peroxidación lipídica en la corteza renal, representado por el aumento en la producción de TBARS. El estado prooxidante en estas células se caracteriza por un incremento en la producción de especies reactivas de oxígeno, situación ante la cual la SOD dismuta el anión superóxido a peróxido de hidrógeno y oxígeno molecular con constante de velocidad alta $\left(2,3.10^{9} \mathrm{M}^{-1} \mathrm{~s}^{-1}\right)$, mientras que la CAT convierte peróxido de hidrógeno en oxígeno molecular y agua.

Era esperable que el aumento de la producción de TBARS se acompañara del aumento de la actividad y/o expresión de SOD y CAT. Pero en nuestros modelos estos parámetros no se vieron afectados. Sin embargo, se registró aumento de la actividad de la GPx, que sugiere un efecto compensatorio a la ausencia de modificaciones de SOD y CAT. La regulación de su actividad está relacionada con modificaciones postraduccionales que tienen lugar en el sitio activo de la enzima y que ocurren independientemente de que varíe o no, su expresión. (38)

\section{CONCLUSIÓN}

Estos resultados sugieren que el anión cloruro es corresponsable, junto con el sodio, para desencadenar daño oxidativo renal e incrementar la presión arterial; de ahí la importancia de tener en cuenta la reducción en la ingesta de ambos iones como medida de tratamiento no farmacológico de la hipertensión arterial, considerando que la mayoría de los productos dietéticos comerciales, sustitutos de la sal de mesa, son a base de cloruro de potasio.

\section{Consideraciones éticas}

El protocolo utilizado fue aprobado por el Comité Institucional para el Cuidado y Uso de Animales de Laboratorio de la Facultad de Farmacia y Bioquímica de la Universidad de Buenos Aires (CICUAL), por $\operatorname{Res}(D) \mathrm{N}^{\circ} 1881 / 2019$, y los procedimientos fueron realizados siguiendo las indicaciones de la "Guía para el cuidado y uso de animales de laboratorio" perteneciente a la Academia Nacional de los Estados Unidos de Norteamérica.

\section{Agradecimientos}

Agradecemos a la Técnica Cecilia Mambrín, de la Cátedra de Nutrición de la Facultad de Farmacia y Bioquímica, Universidad de Buenos Aires, por la preparación de las dietas empleadas en este proyecto.

\section{BIBLIOGRAFÍA}

1. Kurtz TW, Al-Bander HA, Morris RC. 'Salt-sensitive' essential hypertension in men. Is the sodium ion alone important? N. Engl J Med 1987;317:1043-8. https://doi.org/10.1056/NEJM198710223171702

2. Luft FC, Zemel MB, Sowers JA, Fineberg NS, Weinberger MH. Sodium bicarbonate and sodium chloride: effects on blood pressure and electrolyte homeostasis in normal and hypertensive man. J Hypertens 1990;8:663-70. https://doi.org/10.1097/00004872-199007000-00010

3. Shore AC, Markandu ND, MacGregor GA. A randomized crossover study to compare the blood pressure response to sodium loading with and without chloride in patients with essential hypertension. J Hypertens 1988;6:613-7. https://doi.org/10.1097/00004872198808000-00003

4. Berghoff RS, Geraci AS. The influence of sodium chloride on blood pressure. BMJ 1929;56:395-7.

5. van der Leeuw J, Martin H de Borst MH, Kieneker LM, Bakker SJL, Gansevoort RT, Rookmaaker MB. Separating the effects of 24-hour urinary chloride and sodium excretion on blood pressure and risk of hypertension: Results from PREVEND. PLoS One 2020;15:e228490. https://doi.org/10.1371/journal.pone.022849

6. Zanfirescu A, Ungurianu A, Tsatsakis AM, Nitulescu GM, Kouretas D, Veskoukis, et al. A review of the alleged health hazards of monosodium glutamate. Compr Rev Food Sci Food Saf 2019;18:1111-34. https://doi.org/10.1111/1541-4337.12448

7. Iqbal S, Klammer N, Ekmekcioglu C. The Effect of Electrolytes on Blood Pressure: A Brief Summary of Meta-Analyses. Nutrients 2019;11:1362. https://doi.org/10.3390/nu11061362

8. Robinson AT, Edwards DG, Farquhar WB. The Influence of Dietary Salt Beyond Blood Pressure. Curr Hypertens Rep 2019;21:42. https:// doi.org/10.1007/s11906-019-0948-5

9. Jacques T, Picard N, Lance Miller R, Riemondy KA, Houillier P, Sohet F, et al. Overexpression of pendrin in intercalated cells produces chloride-sensitive hypertension. JASN 2013;24:1104-13. https:/doi. org/10.1681/ASN.2012080787

10. Rosón MI, Cavallero S, Della Penna S, Cao G, Gorzalczany S, Pandolfo M, et al. Acute sodium overload produces renal tubulointerstitial inflammation in normal rats. Kidney Int 2006;70:1439-46. https://doi.org/10.1038/sj.ki.5001831

11. Della Penna SL, Cao G, Kouyoumdzian NM, Sarati L, Fellet A, Balaszczuk AM, et al. Role of angiotensin II and oxidative stress on renal aquaporins expression in hypernatremic rats. J Physiol Biochem 2014;70:465-78. https://doi.org/10.1007/s13105-014-0324-5

12. Rosón MI, Cao G, Della Penna S, Gorzalczany S, Pandolfo M, Medici C, et al. Sodium load combined with low doses of exogenous angiotensin II upregulate intrarenal angiotensin II. Kidney Blood Press Res 2009; 32:334-41. https://doi.org/10.1159/000245036

13. Rosón MI, Della Penna SL, Cao G, Gorzalczany S, Pandolfo M, Cerrudo C, et al. High-sodium diet promotes a profibrogenic reaction in normal rat kidneys: effects of Tempol administration. J. Nephrol 2011;24:119-27. https://doi.org/10.5301/JN.2010.5824

14. Rosón MI, Cao G, Della Penna SL, Gorzalczany S, Pandolfo M, Toblli JE, et al. Angiotensin II increases intrarenal transforming growth factor-beta1 in rats submitted to sodium overload independently of blood pressure. Hypertens Res Off Jpn Soc Hypertens 2008;31:707-15. https://doi.org/10.1291/hypres.31.707

15. Kobori, H. \& Nishiyama, A. Effects of tempol on renal angiotensinogen production in Dahl salt-sensitive rats. Biochem Biophys Res Commun 2004;315:746-50.https://doi.org/10.1016/j.bbrc.2004.01.120 16. Della Penna SL, Rosón MI, Toblli JE, Fernández BE. Role of angiotensin II and oxidative stress in renal inflammation by hypernatremia: benefits of atrial natriuretic peptide, losartan, and tempol. Free Radic Res 2015;49:383-96. https://doi.org/10.3109/10715762.2015.1006216 17. Reeves PG, Nielsen FH, Fahey GC Jr. AIN-93 Purified Diets for Laboratory Rodents: Final Report of the American Institute of Nutrition Ad Hoc Writing Committee on the Reformulation of the AIN-76A Rodent Diet. J Nutr 1993;123: 1939-51. https://doi. org/10.1093/jn/123.11.1939 
18. Lowry OH, Rosebrough NJ, Farr AL, Randall RJ. Protein measurement with the Folin phenol reagent. J Biol Chem 1951;193:265-75. https://doi.org/10.1016/S0021-9258(19)52451-6

19. Fraga CG, Leibovitz BE, Tappel AL. Halogenated compounds as inducers of lipid peroxidation in tissue slices. Free Radic Biol Med 1987;3:119-23. https://doi.org/10.1016/S0891-5849(87)80006-0

20. Boveris A, Fraga CG, Varsavsky AI, Koch OR. Increased chemiluminescence and superoxide production in the liver of chronically ethanol-treated rats. Arch Biochem Biophys 1983;227:534-41. https:// doi.org/10.1016/0003-9861(83)90482-4

21. Aebi H. Catalase in vitro. Methods Enzymol 1984;105:121-6. https://doi.org/10.1016/S0076-6879(84)05016-3.

22. Chance B, Sies H, Boveris A. Hydroperoxide metabolism in mammalian organs. Physiol Rev 1979;59:527-605. https://doi.org/10.1152/ physrev.1979.59.3.527

23. Flohé L, Günzler WA. Assays of glutathione peroxidase. Methods Enzymol 1984;105:114-21. https://doi.org/10.1016/S00766879(84)05015-1

24. Kouyoumdzian NM, Rukavina Mikusic NL, Robbesaul GD, Gorzalczany SB, Carranza A, Trida V. Acute infusion of angiotensin II regulates organic cation transporters function in the kidney: its impact on the renal dopaminergic system and sodium excretion. Hypertens Res 2020;44:286-98. https://doi.org/10.1038/s41440-020-00552-7

25. Cui H, Yang S, Zheng M, Liu R, Zhao G, Wen J. High-salt intake negatively regulates fat deposition in mouse. Sci Rep 2017;7:2053. https://doi.org/10.1038/s41598-017-01560-3

26. Kouyoumdzian NM, Rukavina Mikusic NL, Cao G, Choi MR, Della Penna SL, Fernández BE, et al. Adverse effects of tempol on hidrosaline balance in rats with acute sodium overload. Biotech Histochem 2016;91:510-21. https://doi.org/10.1080/10520295.2016.1249029 27. Cao G, Della Penna SL, Kouyoumdzian NM, Choi MR, Gorzalczany $\mathrm{S}$, Fernández BE, et al. Immunohistochemical expression of intrarenal renin angiotensin system components in response to tempol in rats fed a high salt diet. World J Nephrol 2017;6:29-40. https://doi. org/10.5527/wjn.v6.i1.29

28. McCallum L, Lip S, Padmanabhan S. The hidden hand of chloride in hypertension. Pflugers Arch 2015;467:595-603. https://doi. org/10.1007/s00424-015-1690-8

29. Abboud HE, Luke RG, Galla JH, Kotchen TA. Stimulation of renin by acute selective chloride depletion in the rat. Circ Res 1979;44:815-21. https://doi.org/10.1161/01.RES.44.6.815

30. Kotchen TA, Galla JH, Luke RG. Failure of NaHCO3 and KHCO3 to inhibit renin in the rat. Am J Physiol 1976;231:1050-6. https://doi. org/10.1152/ajplegacy.1976.231.4.1050

31. Kotchen TA, Luke RG, Ott CE, Galla JH, Whitescarver S. Effect of chloride on renin and blood pressure responses to sodium chloride. Ann Intern Med 1983;98: 817-22. https://doi.org/10.7326/0003-4819-98-5-817 32. Machnik A, Dahlmann A, Kopp C, Goss J, Wagner H, van Rooijen $\mathrm{N}$, et al. Mononuclear phagocyte system depletion blocks interstitial tonicity-responsive enhancer binding protein/vascular endothelial growth factor $\mathrm{C}$ expression and induces salt-sensitive hypertension in rats. Hypertension 2010;55:755-61. https://doi.org/10.1161/HYPERTENSIONAHA.109.143339

33. Machnik A, Neuhofer W, Jantsch J, Dahlmann A, Tammela T, Machura K, et al. Macrophages regulate salt-dependent volume and blood pressure by a vascular endothelial growth factor-Cdependent buffering mechanism. Nat Med 2009;15: 545-52. https:// doi.org/10.1038/nm.1960

34. Matsuda JJ, Filali MS, Moreland JG, Miller FJ, Lamb FS. Activation of swelling-activated chloride current by tumor necrosis factor-alpha requires ClC-3-dependent endosomal reactive oxygen production. J Biol Chem 2010;285:22864-73. https://doi.org/10.1074/ jbc.M109.099838

35. Di Ciano LA, Azurmendi PJ, Vlachovsky SG, Celía AF, Oddo EM, Arrizurieta EE, et al. Gender differences in blood pressure, renal function and response to high-sodium diet in Wistar rats. Nefrol Dial Traspl 2018;38:15-27. https://doi.org/10.1016/j.lfs.2012.04.011 36. Schmidlin O, Tanaka M, Bollen AW, Yi SL, Morris RC Jr. Chloridedominant salt sensitivity in the stroke-prone spontaneously hypertensive rat. Hypertension 2005;45:867-73. https://doi.org/10.1161/01. HYP.0000164628.46415.66

37. Schmidlin O, Tanaka M, Sebastian A, Morris RC Jr. Selective chloride loading is pressor in the stroke-prone spontaneously hypertensive rat despite hydrochlorothiazide-induced natriuresis. J Hypertens 2010;28:87-94. https://doi.org/10.1097/HJH.0b013e3283316cfc

38. Cao C, Leng Y, Huang W, Liu X, Kufe D. Glutathione peroxidase 1 is regulated by the c-Abl and Arg tyrosine kinases, J Biol Chem 2003;278:39609-14. https://doi.org/10.1074/jbc.M305770200 